\title{
Refluxo gastroesofágico em cadelas durante ovário-histerectomia convencional submetidas a diferentes medicações pré-anestésicas
}

\author{
[Gastroesophageal reflux in dogs undergoing conventional ovariohysterectomy \\ using different premedications] \\ H.F. Hartmann ${ }^{1}$, J.P.S. Feranti ${ }^{1}$, M.T. Oliveira ${ }^{1}$, M.T. Linhares ${ }^{1}$, L.F.D. Correa ${ }^{1}$, \\ G.P. Coradini ${ }^{1}$, B. Pires ${ }^{1}$, M.V. Brun ${ }^{1,2^{*}}$ \\ ${ }^{1}$ Universidade Federal de Santa Maria - UFSM - Santa Maria, RS \\ ${ }^{2}$ Bolsista de produtividade do $\mathrm{CNPq}$ \\ RESUMO
}

\begin{abstract}
A doença do refluxo gastroesofágico decorre do fluxo de conteúdo gastroduodenal para o esôfago e/ou para os órgãos adjacentes, o que leva à ampla gama de sinais e implicações clínicas. É desconhecida a incidência de refluxo gastroesofágico transoperatório em caninos. O objetivo deste trabalho foi, por meio da endoscopia flexível, avaliar a presença do refluxo gastroesofágico em cadelas submetidas a ováriohisterectomia com base nos fármacos analgésicos utilizados na medicação pré-anestésica (morfina, tramadol ou metadona). Concluiu-se que não houve diferença na incidência de refluxo gastroesofágico transoperatório, não tendo os fármacos testados influenciado de forma diferente esse comportamento; porém, alguns animais do grupo morfina apresentaram êmese pré-operatória. A gravidade dos refluxos foi maior nas cadelas submetidas ao uso da metadona, de acordo com o método de avaliação utilizado para esta pesquisa.
\end{abstract}

Palavras-chave: endoscopia, regurgitação transoperatória, pré-medicação, canino

\begin{abstract}
Gastroesophageal reflux disease arises from the gastroduodenal content flow to the esophagus and/or associated organs, which leads to the wide range of signs and clinical implications. Incidence of intraoperative gastroesophageal reflux disease in dogs is unknown. The objective was, through flexible endoscopy, to assess the presence of gastroesophageal reflux in dogs undergoing ovariohysterectomy based on analgesic drugs used in premedication (morphine, tramadol or methadone). It was concluded that there was no difference in the incidence of intraoperative gastroesophageal reflux, these drugs don't influence this behavior; however, in the morphine group some animals showed preoperative vomiting. The severity of the reflux was higher, but not significantly, in bitches undergoing the methadone according to the evaluator method used for this research.
\end{abstract}

Keywords:endoscopy, intraoperative regurgitation, surgery, premedication, canine

\section{INTRODUÇÃO}

O refluxo gastroesofágico (RGE) é definido como o fluxo de conteúdo gastroduodenal para o esôfago e adjacências, que desencadeia vários sinais clínicos (Norton e Pena, 2000; Barczinski e Moraes-Filho, 2006; Carvalhaes et al., 2011; Abrahão Jr., 2014). Várias espécies são acometidas por esse evento e diversas podem ser as etiologias (Favarato et al., 2010).
Em cães, a anestesia geral é uma das principais causas de esofagite, devido à redução do tônus esfíncter esofagogástrico, precipitada por alguns fármacos (Favarato et al., 2010). O RGE pode incorrer em algumas consequências, tais como: azia, regurgitação, esofagite (Münster et al., 2013); pneumonia aspirativa (Nogueira et al., 2003) e estenose esofágica (Vlasin et al., 2004; Silva et al., 2010; Oliveira et al., 2013). O RGE em humanos ainda está fortemente associado ao

Recebido em 27 de outubro de 2016

Aceito em 3 de dezembro de 2016

* Autor para correspondência (corresponding author)

E-mail: mauriciovelosobrun@hotmail.com 
risco de desenvolvimento de adenocarcinoma de esôfago (Lagergren et al., 1999).

Um índice de 5\% de RGE transoperatório tem sido reportado em humanos, sendo esse refluxo inaparente e geralmente ácido $(\mathrm{Ng}$ e Smith, 2001). Quando o conteúdo refluído atinge a faringe, é considerado regurgitação e pode ser aspirado pelo pulmão (Wilson et al., 2005), o que resulta em maiores taxas de morbidade e mortalidade pós-anestésicas (Nogueira et al., 2003).

Em humanos, a injúria péptica, como resultado de exposição crônica da mucosa esofágica, é a causa mais comum de estenoses esofágicas, constituindo um problema frequentemente encontrado na prática endoscópica (Siersema e De Wijkerslooth, 2009). Estenoses, na medicina veterinária, ocorrem mais comumente devido a RGE perianestésico (Lam et al., 2013). Embora estenose esofágica possa se desenvolver a partir de qualquer lesão grave à mucosa, observa-se que a maioria dos casos ocorre por esofagite de refluxo perianestésico, especialmente em fêmeas submetidas a ovário-histerectomia (AdamamaMoraitou et al., 2002).

Ovário-histerectomia e ovariectomia são realizadas em cadelas e gatas com o objetivo de impedir a reprodução ou de tratar afecções como cistos ovarianos, torção ou prolapso uterinos e piometra (Schiochet et al., 2007). Sua prática ainda elimina o risco de falsa gestação (Martins e Lopes, 2005), bem como reduz o risco de neoplasias mamárias (Fonseca e Dalek, 2000), além de ser terapia adjuvante no tratamento de epilepsia (Stone, 1998) e diabetes melito (Pöppl, 2012).

O objetivo do presente trabalho foi comparar a influência de diferentes fármacos pré-anestésicos sobre o refluxo gastroesofágico transoperatório em cadelas, uma vez que são escassos na medicina veterinária os estudos referentes a esse evento. Para tanto e a fim de padronizar os animais estudados, optou-se por cadelas a serem submetidas a ovário-histerectomia eletiva convencional.

\section{MATERIAL E MÉTODOS}

Foram atendidas 29 cadelas, adultas, hígidas, sem histórico de êmese, pesando $11,5 \pm 4,1 \mathrm{~kg}$, com indicação de ovário-histerectomia eletiva. Após aptidão confirmada por exames clínico e laboratorial (hemograma e bioquímica sérica), foram aleatoriamente separadas em três grupos. Um grupo com 10 animais (GI) foi submetido à ovário-histerectomia por celiotomia e recebeu como medicação pré-anestésica (MPA) acepromazina $\left(0,05 \mathrm{mg} \cdot \mathrm{kg}^{-1}\right)$ e morfina $\left(0,5 \mathrm{mg} \cdot \mathrm{kg}^{-1}\right)$ por via intramuscular; outro grupo com 10 animais (GII) foi submetido à mesma técnica operatória e recebeu como MPA acepromazina $\left(0,05 \mathrm{mg} \cdot \mathrm{kg}^{-1}\right)$ e tramadol $\left(4 \mathrm{mg} \cdot \mathrm{kg}^{-}\right.$ ${ }^{1}$ ); e um terceiro grupo, com nove animais (GIII), também submetido à mesma técnica operatória, recebeu como acepromazina $\left(0,03 \mathrm{mg} \cdot \mathrm{kg}^{-1}\right)$ e metadona $\left(0,3 \mathrm{mg} \cdot \mathrm{kg}^{-1}\right)$. Todos os animais tiveram o mesmo período de jejum préoperatório, sob internação hospitalar, de 12 horas de privação alimentar e oito horas de privação hídrica. A sequência do procedimento anestésico ocorreu da mesma forma para todos os grupos: administração de propofol $\left(4 \mathrm{mg} \cdot \mathrm{kg}^{-1}\right)$ para indução anestésica e isoflurano, vaporizado em oxigênio a 100\%, para manutenção anestésica por meio de intubação orotraqueal.

O endoscópio flexível de 9,8mm foi introduzido no esôfago das cadelas até aproximadamente a altura do nono espaço intercostal - mensurado externamente nas cadelas a fim de se observar diretamente a transição esofagogástrica durante o procedimento. Esse posicionamento foi realizado simultaneamente à colocação dos campos operatórios. A ocorrência de refluxo gastroesofágico foi sendo monitorada desde então.

O grau de refluxo gastroesofágico foi classificado conforme a legenda: R0 - sem refluxo, esôfago limpo; R1 - sem refluxo, com bolhas desde o início da esofagoscopia, indicativo de peristaltismo pré-operatório; R2 sem refluxo, com formação de bolhas no transoperatório, indicando um aumento de peristaltismo transoperatório; R3 - refluxo discreto (alcançando a altura do terço distal do esôfago); R4 - refluxo discreto com prolapso de mucosa gástrica para o esôfago; R5 refluxo moderado (atingindo terço médio do esôfago); R6 - refluxo moderado com prolapso de mucosa 
gástrica; R7 - refluxo grave (atingindo terço cranial do esôfago); e R8 - refluxo grave com prolapso de mucosa gástrica. Essa escala foi desenvolvida pelos autores especialmente para o projeto que deu origem a este trabalho.

O projeto que deu origem a este trabalho foi aprovado pelo Comitê de Ética no Uso de Animais (Ceua) - UFSM, sob o parecer 136/2014. Data de aprovação: 18/12/2014.

\section{RESULTADOS E DISCUSSÃO}

O presente estudo contribuiu para o controle populacional de cães errantes no município de Santa Maria e, assim, auxiliou a comunidade, já que os pacientes eram oriundos de organizações não governamentais ou de tutores com baixo poder aquisitivo e, com o estudo, puderam ser esterilizados a um custo muito reduzido.

Em relação ao pré-operatório, via de regra, é solicitado jejum alimentar de 12 horas considerando-se alterações conforme idade do paciente e procedimento cirúrgico a ser submetido (Muir, 2007). Para o presente trabalho, foram selecionadas cadelas hígidas, sem histórico de vômitos ou regurgitações, sendo alimentadas até 12 horas anteriores ao procedimento, quando foram internadas para garantir a realização do jejum. Assim, ficou impossibilitado o acesso à alimentação líquida ou sólida após o período definido, uma vez que, do contrário, poderia haver interferências diretas nos resultados de vômitos e/ou regurgitações.

A dose da acepromazina foi menor quando utilizada em combinação com a metadona do que quando combinada aos outros analgésicos, em razão do maior efeito sedativo dela (Maiante et al., 2009), bem como da proposta de mimetizar as doses utilizadas na rotina hospitalar.

Valverde et al. (1989) demonstraram que administrar a acepromazina 15 minutos antes do opioide reduz a incidência de vômitos induzidos por essas substâncias, porém essa não é uma prática do meio veterinário e deve ser considerada como manobra a ser incorporada na rotina anestésica. Das cadelas pré-medicadas com morfina (GI), sete $(70 \%)$ apresentaram êmese no período pré-operatório, após a administração da medicação pré-anestésica. Das cadelas pré-medicadas com tramadol (GII) e com metadona (GIII), nenhuma apresentou êmese pré-operatória. Wilson et al. (2005) referiram que a êmese causada no pré-operatório não é condição determinante para o refluxo gastroesofágico transoperatório, embora a morfina já tenha sido descrita como fármaco precipitador desse evento (Barczinski e MoraesFilho, 2006). Paolozzi et al. (2011) observaram elevada incidência $(50 \%)$ de êmese nas cadelas que receberam $4 \mathrm{mg} . \mathrm{kg}^{-1}$ de tramadol, porém essas o receberam por via intravenosa. A metadona apresenta vantagem em relação aos outros opioides, como morfina e hidromorfona, por não provocar êmese em cães (Valverde et al., 1989; Maiante et al., 2009).

Para avaliar o refluxo gastroesofágico transoperatório, existem alguns métodos: pHmetria esofágica, monitorização da bilirrubina no lúmen esofágico, impedância intraluminal multicanal e avaliação endoscópica (Favarato et al., 2010). No presente trabalho, optou-se pela avaliação endoscópica, técnica que também permitiria avaliação de anormalidades esofagianas e tomada de amostras para biopsias.

No transoperatório, das cadelas do GI, três apresentaram refluxo gastroesofágico classificado em R3. Duas cadelas manifestaram R1 e duas manifestaram R2; estas duas classificações não foram consideradas efetivamente refluxo, por não se enquadrarem na definição de Norton e Pena (2000), Barczinski e Moraes-Filho (2006), Carvalhaes et al. (2011) e Abrahão Jr. (2014) para essa condição. Considerou-se que um peristaltismo esofágico saliente no período pré-operatório justificaria a presença de bolhas ou espuma já no início da endoscopia e que um peristaltismo transoperatório justificaria a formação de bolhas durante o procedimento, sendo, talvez, uma fase prévia do refluxo propriamente dito. Entre as cadelas do GII, três apresentaram refluxo gastroesofágico classificado em R3, uma em R2 e três em R1. Já no GIII, três cadelas apresentaram refluxo gastroesofágico classificado em R5, e todas as demais em R1. De acordo com a pesquisa de Favarato et al. (2010), quando a morfina foi utilizada, o índice de refluxo gastroesofágico transoperatório nos cães variou entre 46,6 e $63,3 \%$, e quando a metadona foi utilizada, esse índice foi de $52 \%$. Essa variação na incidência de refluxo com a morfina foi devido à utilização de diferentes agentes 
indutores, nenhum deles sendo o propofol. Já o protocolo com metadona foi semelhante ao utilizado no presente estudo - associado à acepromazina na pré-medicação, seguido de propofol e isoflurano.

Entre os três grupos aqui estudados, $30 \%$ dos animais apresentaram refluxo gastroesofágico transoperatório no GI e no GII e 33,3\% dos animais manifestaram refluxo no GIII. Como representado na Tab. 1, não existe associação entre o tipo de fármaco analgésico utilizado na medicação pré-anestésica empregada (na posologia testada) e o refluxo gastroesofágico $(\mathrm{P}=0,98)$, em nível de $5 \%$ de significância pelo teste qui-quadrado.

Tabela 1. Representação da incidência de refluxo gastroesofágico transoperatório em cadelas submetidas à ovário-histerectomia convencional conforme os fármacos analgésicos utilizados na pré-medicação anestésica. Não existe associação entre o tipo de fármaco analgésico utilizado e o refluxo gastroesofágico transoperatório $(\mathrm{P}=0,98)$, em nível de $5 \%$ de significância pelo teste qui-quadrado

\begin{tabular}{lllll}
\hline & \multicolumn{4}{c}{ Grupos quanto aos analgésicos* } \\
\hline Refluxo gastroesofágico & Grupo I & Grupo II & Grupo III & Total \\
Sim & $3(30,0 \%)$ & $3(30,0 \%)$ & $3(33,3 \%)$ & $9(31,03 \%)$ \\
Tão & $7(70,0 \%)$ & $7(70,0 \%)$ & $6(66,7 \%)$ & $20(68,97 \%)$ \\
\hline Total & $10(100 \%)$ & $10(100 \%)$ & $9(100 \%)$ & 29 \\
\hline
\end{tabular}

*Grupo I: morfina; grupo II: tramadol; grupo III: metadona.

A critério comparativo, no GIII os refluxos registrados foram mais graves. Enquanto nos dois primeiros grupos os refluxos mais severos foram classificados em R3, aquele em que o conteúdo atinge apenas o terço distal do esôfago, no último grupo os registros chegaram a ser classificados em R5, aquele em que o conteúdo atinge o terço médio do esôfago. Não foram encontradas na literatura veterinária avaliações de intensidade de refluxo gastroesofágico realizadas por endoscopia. Em medicina humana, rotineiramente a intensidade é avaliada por pHmetria (Nasi et al., 2006).

Foi observado por Panti et al. (2009) que, em cães, a administração de omeprazol algumas horas antes do procedimento anestésico reduziu a incidência de refluxo gastroesofágico. Esse pode ser um procedimento a ser adotado naqueles pacientes sob risco de refluxo (quer seja pelo tipo de procedimento a ser submetido, quer seja pela medicação utilizada ou pela condição clínica), visto que a redução da acidez gástrica parece aumentar a competência do esfíncter esofagogástrico (Ng e Smith, 2001).

Todos os animais tiveram os refluxos aspirados como preconizado por Hall (2015). As cadelas que manifestaram refluxo tiveram a prescrição pós-operatória de omeprazol $\left(1 \mathrm{mg} \cdot \mathrm{kg}^{-1}\right)$ via oral, a cada $24 \mathrm{~h}$, durante cinco dias. Tal tratamento consistiu em reduzir a acidez gástrica, prevenir refluxos subsequentes e proteger a mucosa esofágica (Nelson e Couto, 2010). Nenhum animal que participou deste trabalho teve qualquer manifestação clínica referente aos procedimentos realizados num período mínimo de 16 e máximo de 40 semanas, na dependência da data de realização da ovário-histerectomia.

\section{CONCLUSÃO}

Não houve diferença na incidência de refluxo gastroesofágico transoperatório entre os grupos conforme os protocolos pré-anestésicos utilizados. A êmese pré-operatória não influenciou no refluxo gastroesofágico transoperatório.

\section{REFERÊNCIAS}

ABRAHÃO JR, L.J. Doença do refluxo gastroesofágico. J. Bras. Med., v.102, p.31-36, 2014.

ADAMAMA-MORAITOU, K.K.; RALLIS, T.S.; PRASSINOS, N.N.; GALATOS, A.D. Benign esophageal stricture in the dog and cat: a retrospective study of 20 cases. Can. J. Vet. Res., v.66, p.55-59, 2002.

BARCZINSKI, T.; MORAES-FILHO, J.P.P. Doença do refluxo gastroesofágico na mulher. Rev. Bras. Med., v.63, p.160-168, 2006.

CARVALHAES, A.; FERRARI JR, A.P.; MAGALHÃES, A.F. et al. Doença do refluxo gastroesofágico: diagnóstico. Rev. Assoc. Med. Bras., v.57, p.499-507, 2011. 
FAVARATO, E.S.; SOUZA, M.V.; COSTA, P.R.S. Refluxo gastroesofágico em cães anestesiados: fisiopatologia, clínica, diagnóstico e terapêutica. Ciênc. Rural, v.40, p.2427-2434, 2010.

FONSECA, C.S.; DALECK, C.R. Neoplasias mamárias em cadelas: influência hormonal e efeitos da ováriohisterectomia como terapia adjuvante. Ciênc. Rural, v.30, p.731-735, 2000.

HALL, E. Endoscopy of the gastrointestinal tract in dogs and cats. In Pract., v.37, p.155-168, 2015.

LAGERGREN, J.; BERGSTRÖM, R.; LINDGREN, A.; NYRÉN, O. Symptomatic gastroesophageal reflux as a risk factor for esophageal adenocarcinoma. $N$. Engl. J. Med., v.340, p.825-831, 1999.

LAM, N.; WEISSE, C.; BERENT, A. et al. Esophageal stenting for treatment of refractory benign esophageal strictures in dogs. J. Vet. Intern. Med., v.27, p.1064-1070, 2013.

MAIANTE, A.A.; TEIXEIRA NETO, F.J.; BEIER, S.L. et al. Comparison of the cardio-respiratory effects of methadone and morphine in conscious dogs. J. Vet. Pharmacol. Ther., v.32, p.317-328, 2009.

MARTINS, L.R.; LOPES, M.D. Pseudociese canina. Rev. Bras. Reprod. Anim., v.29, p.137-141, 2005.

MUIR, W.W. Considerations for general anesthesia. In: TRANQUILI, W.J. et al. Lumb \& Jones' veterinary anesthesia and analgesia. 4.ed. Iowa: Blackwell Publishing, 2007. p.7-30.

MÜNSTER, M.; VIETH, M.; HÖRAUF, A. Evaluation of the quality of endoscopically obtained esophageal biopsies in the dog. Tierarztl. Prax. Ausg. K Kleintiere Heimtiere, v.41, p.375-382, 2013.

NASI, A.; MORAES-FILHO, J.P.P.; CECCONELLO, I. Doença do refluxo gastroesofágico: revisão ampliada. Arq. Gastroenterol., v.43, p.334-341, 2006.

NELSON, R.W.; COUTO, C.G. Doenças da cavidade oral, da faringe e do esôfago. In: Medicina interna de pequenos animais. 4.ed. Rio de Janeiro: Elsevier, 2010. cap.31, p.414-425.

NG, A.; SMITH, G. Gastroesophageal reflux and aspiration of gastric contents in anesthetic practice. Anesthesiol. Analg., v.93, p.494-513, 2001.

NOGUEIRA, L.C.; CORTOPASSI, S.R.G.; INTELIZANO, T.R.; SOUZA, M.S.B. Efeitos do jejum alimentar pré-cirúrgico sobre a glicemia e o período de recuperação anestésica em cães. Braz. J. Vet. Res. Anim. Sci., v.40, Supl.1, p.20-25, 2003.
NORTON, R.C.; PENNA, F.J. Refluxo gastroesofágico. J. Pedriatr., v.76, Supl.2, p.218-224, 2000.

PANTI, A.; BENNETT, R.C.; CORLETTO, F. et al. The effect of omeprazole on oesophageal $\mathrm{pH}$ in dogs during anaesthesia. J. Small Anim. Pract., v.50, p.540544, 2009.

PAOLOZZI, R.J.; CASSU, R.N.; DA CRUZ, F.S.F.; PARRILHA, R.L. Diferentes doses de tramadol em cães: ações analgésicas, sedativas e sobre o sistema cardiorrespiratório. Ciênc. Rural, v.41, n.8, p.14171423, 2011

OLIVEIRA, M.T.; TRINDADE, A.B.; DE SOUZA, F.W. et al. Dilatação esofágica endoscópica associada ao uso de triancinolona intramural em cadela com estenose de esôfago após ovariohisterectomia eletiva. Ciênc. Rural, v.43, p.1683-1686, 2013.

PÖPPL, A.G. Estudos clínicos sobre os fatores de risco e a resistência à insulina na diabetes mellitus em cães. 2012. 215f. Tese (Doutorado em Ciências Veterinárias) - Programa de Pós-graduação em Ciências Veterinárias, Universidade Federal do Rio Grande do Sul, Porto Alegre, RS.

SCHIOCHET, F.; BECK, C.A.C.; PINTO, V. et al. Ovariectomia laparoscópica em uma gata com ovários remanescentes. Acta Sci. Vet., v.35, p.245-248, 2007.

SIERSEMA, P.D.; WIJKERSLOOTH, L.R.H. Dilation of refractory benign esophageal stricture. Gastrointest. Endosc., v.70, p.1000-1012, 2009.

SILVA, E.C.S.; PINA, F.L.S.; TEIXEIRA, M.W. Diagnóstico e tratamento da estenose esofágica pela via endoscópica em cão: relato de caso. Cienc. Anim. Bras., v.11, p.465-470, 2010.

STONE, E.A. Ovário e útero. In: SLATTER, D. Manual de cirurgia de pequenos animais. 2.ed. São Paulo: Manole, 1998, p.1540-1558.

VALVERDE, A.; DYSON, D.H.; MC DONNEL, W.N. Epidural morphine reduces halothane MAC in the dog. Can. J. Anaesthesiol., v.36, p.629-632, 1989.

VLASIN, M.; HUSNIK, R.; FICHTEL, T.; RAUSEROVÁ, L. Acquired esophageal stricture in the dog: a case report. Vet. Med. Czech J., v.49, p.143147, 2004.

WILSON, D.V.; EVANS, A.T.; MILLER, R. Effects of preanesthetic administration of morphine on gastroesophageal reflux and regurgitation during anesthesia in dogs. Am. J. Vet. Res., v.66, p.386-390, 2005. 